\title{
Low-noise mid-IR upconversion detector for improved IR-degenerate four-wave mixing gas sensing
}

Høgstedt, Lasse; Dam, Jeppe Seidelin; Sahlberg, Anna-Lena; Li, Zhongshan; Alden, Marcus; Pedersen, Christian; Tidemand-Lichtenberg, Peter

Published in:

Optics Letters

Link to article, DOI:

10.1364/OL.39.005321

Publication date:

2014

Document Version

Publisher's PDF, also known as Version of record

Link back to DTU Orbit

Citation (APA):

Høgstedt, L., Dam, J. S., Sahlberg, A-L., Li, Z., Alden, M., Pedersen, C., \& Tidemand-Lichtenberg, P. (2014). Low-noise mid-IR upconversion detector for improved IR-degenerate four-wave mixing gas sensing. Optics Letters, 39(18), 5321-5324. https://doi.org/10.1364/OL.39.005321

\section{General rights}

Copyright and moral rights for the publications made accessible in the public portal are retained by the authors and/or other copyright owners and it is a condition of accessing publications that users recognise and abide by the legal requirements associated with these rights.

- Users may download and print one copy of any publication from the public portal for the purpose of private study or research.

- You may not further distribute the material or use it for any profit-making activity or commercial gain

- You may freely distribute the URL identifying the publication in the public portal 


\title{
Low-noise mid-IR upconversion detector for improved IR-degenerate four-wave mixing gas sensing
}

\author{
Lasse Høgstedt, ${ }^{1, *}$ Jeppe Seidelin Dam, ${ }^{1}$ Anna-Lena Sahlberg, ${ }^{2}$ Zhongshan $\mathrm{Li}^{2}{ }^{2}$ \\ Marcus Aldén, ${ }^{2}$ Christian Pedersen, ${ }^{1}$ and Peter Tidemand-Lichtenberg ${ }^{1}$ \\ ${ }^{1}$ Department of Photonics Engineering, Technical University of Denmark, Frederiksborgvej 399, 4000 Roskilde, Denmark \\ ${ }^{2}$ Department of Physics, Combustion Physics, Lund University, Professorsgatan 1, SE-223 63 Lund, Sweden \\ ${ }^{*}$ Corresponding author: lhog@fotonik.dtu.dk
}

Received June 10, 2014; revised July 30, 2014; accepted July 30, 2014;

posted July 31, 2014 (Doc. ID 212856); published September 8, 2014

\begin{abstract}
We compare a nonlinear upconversion detector with a conventional cryogenic InSb detector for the detection of coherent infrared light showing near-shot-noise-limited performance in the upconversion system. The InSb detector is limited by dark noise, which results in a 500 times lower signal-to-noise ratio. The two detectors are compared for the detection of a coherent degenerate four-wave mixing (DFWM) signal in the mid-infrared, and applied to measure trace-level acetylene in a gas flow at atmospheric pressure, probing its fundamental rovibrational transitions. In addition to lower noise, the upconversion system provides image information of the signal, thus adding new functionality compared to standard point detection methods. We further show that the upconversion detector system can be implemented as a simple replacement of the cryogenic detector. (c) 2014 Optical Society of America

OCIS codes: (040.3060) Infrared; (280.4788) Optical sensing and sensors; (280.1740) Combustion diagnostics; (190.7220) Upconversion; (110.3080) Infrared imaging.

http://dx.doi.org/10.1364/OL.39.005321
\end{abstract}

Mid-infrared (mid-IR) detectors are applied in a wide range of applications and in a wide range of fields, from surveillance and analysis in industry to advanced research applications. Most detectors are solid-state devices based on direct detection of mid-IR light [1] and, as a result of inherent thermal radiation, dark counts are often a major noise issue. Alternative methods based on nonlinear upconversion in periodically poled lithium niobate (PPLN) waveguides have been demonstrated around $1550 \mathrm{~nm}[2,3]$ and $2 \mu \mathrm{m}$ [4] with high efficiencies, allowing for the use of low-noise visible light avalanche photodiodes for detection of infrared (IR) light. The same principles have been applied for the upconversion of incoherent mid-IR images by Dam et al. [5] using a continuous wave intracavity PPLN mixing scheme.

Given the widespread use of mid-IR detectors, upconversion detection can have a large impact on areas that work with low-noise mid-IR measurements. The implementation of the upconversion technology will in most setups be a simple detector replacement, using wellestablished silicon technology to detect the upconverted light.

Molecular spectroscopy in the mid-IR spectral range is attracting growing interest, particularly for gas sensing in relation to energy and environmental applications, due to the unique possibility of sensitive measurements of a long list of crucial molecular species, e.g., $\mathrm{C}_{2} \mathrm{H}_{2}, \mathrm{CH}_{4}$, OCS, $\mathrm{H}_{2} \mathrm{~S}, \mathrm{HCl}, \mathrm{HF}$, and $\mathrm{HCN}$, which otherwise can hardly be detected. For this reason, a sensitive and noise-free detection method of signal photons in the mid-IR is of utmost importance.

Spectroscopic methods, such as grating-based monochromators and Fourier transform IR spectroscopy, are commercially available and applied on a routine basis. For pulsed in situ measurements, IR polarization spectroscopy and degenerate four-wave mixing (DFWM) have been used within combustion analysis, and acetylene concentrations down to 30 parts per million (ppm) have been detected [ㅁ,]].
Going to lower concentrations would allow for a more detailed analysis of the combustion processes and, at the sub-ppm and parts-per-billion levels, applications in other fields appear, e.g., breath analysis [8] and explosives detection.

In [9] , a pump-probe experiment demonstrates 2D IR spectroscopy with upconversion using femtosecond pulses and shows that upconversion is feasible for detection of mid-IR signals in DWFM setups. The setup, however, is highly complicated due to the timing issues.

In [10], the combination of upconversion detection and DWFM was first demonstrated in connection with the improved detection of acetylene. These measurements demonstrated detection of acetylene concentrations down to $3 \mathrm{ppm}$. However, no quantitative comparison of detector performance and the (signal-to-noise ratio) SNR of the upconversion-based system was made relative to traditional cryogenically cooled InSb detectors. Using a setup similar to the one in $[5,10]$, this Letter quantifies a 500 times improvement in $\overline{\mathrm{S}} \overline{\mathrm{NR}}$ compared to a conventional cryogenic InSb detector, using continuous wave upconversion detection. Furthermore, a threefold improvement, down to $1 \mathrm{ppm}$ in the detection of acetylene concentration, is demonstrated, compared to previous results reported with a DFWM setup using upconversion [10] and more than a 10 times improvement compared to InSb-based detection. The system is based on nonlinear frequency conversion well known from secondharmonic generation and can be realized in a compact fashion using commercially available components.

For the benchmark test between the upconversion detector at room temperature and the cryogenic InSb detector, DFWM is a suitable setup, as this is, in principle, a background-free technique and the sensitivity in detection of low gas concentrations is limited directly by the detector sensitivity and noise level. In practical measurements, however, there is always unwanted scattered laser light close to the DFWM signal beam. This was also the case in the current setup, but with the imaging 
capabilities of the upconversion detector it was possible to decrease the background level significantly, as mentioned in [10].

The setup used in the experiment is illustrated in Fig. 1 and consists of three sections: the mid-IR light source, the gas tube where the four-wave mixing occurs, and the dual detection section. The light source [ $\underline{6}]$ is based on a $10 \mathrm{~Hz}$ tunable dye laser around $790 \mathrm{~nm}$ that is frequency mixed in a $\mathrm{LiNbO}_{3}$ crystal to provide mid-IR light tunable from 2900 to $3400 \mathrm{~cm}^{-1}$. This is amplified in a second $\mathrm{LiNbO}_{3}$ crystal to achieve pulse energies of 4$5 \mathrm{~mJ}$. The pulse length is around $4 \mathrm{~ns}$ and the linewidth has been measured to be $0.025 \mathrm{~cm}^{-1}$ [11]. The beam is passed through a set of BOXCAR plates [7] and split into four beams of equal intensities. Three of these beams are focused to overlap at the center of the flow cell with an estimated interaction volume of $(0.4 \times 0.4 \times 10) \mathrm{mm}^{3}$. Through DFWM, the three beams will generate a fourth beam when acetylene molecules are present in the interaction volume. This fourth beam constitutes the signal and the residual of the three beams is blocked. The signal beam is then passed on to scaling optics and apertures.

One aperture was placed in the Fourier plane to block high spatial frequency scattering components. A flip mirror directed the signal beam to either the upconversion detector, or the cryogenic InSb detector (J10D-M204R04M-60, Teledyne Judson Technologies) to reproduce the single detector setup [7]. In the upconversion module, the vertically polarized mid-IR signal beam passes through a germanium window and onto a $5 \% \mathrm{MgO}$-doped $\mathrm{LiNbO}_{3}$ crystal placed inside a $70 \mathrm{~W}$ intracavity field at $1064 \mathrm{~nm}$ in a Nd: $\mathrm{YVO}_{4}$ laser. In the PPLN crystal, poled with a period of $22 \mu \mathrm{m}$, the mid-IR signal is frequency mixed with the intracavity field to generate near visible light around $800 \mathrm{~nm}$. This light is passed through a set of filters before it is detected by an electron multiplying CCD chip (Luca, Andor Technology). To avoid stray light from background emissions, the whole upconversion detector system is sealed in a black box. The beam waist of the mixing laser inside the PPLN crystal is $180 \mu \mathrm{m}$, defining the effective detector area. The upconversion module is described in greater detail in [5], the only change being that the pump diode for the $\mathrm{Nd}: \mathrm{YVO}_{4}$ laser has been exchanged with an $880 \mathrm{~nm}$ laser diode to separate the pump wavelength from the visible signal wavelength. This will allow for more efficient filtering and thus minimize the $800 \mathrm{~nm}$ light reaching the CCD chip as spill light from the pump.
A measurement was conducted by recording the signal from the gas flow at a given acetylene concentration while the wavelength of the mid-IR light source was scanned at a rate of $0.05 \mathrm{~cm}^{-1} / \mathrm{s}$. For the InSb detector, the data acquisition is a straightforward oscilloscope reading. The imaging capability of the upconversion detector [5] is utilized in the data acquisition for the upconversion detector, and Fig. 2 shows a raw image of the upconverted mid-IR signal. A full measurement scan consists of a whole range of CCD frames, each assigned to a specific pulse and wavelength. Each point in a spectrum is thus the sum of the data pixels in a single frame. The data pixels are selected for the lowest concentration to optimize the signal/scattering ratio, and this selection is then applied globally to all the measurements. It is not entirely possible to suppress the scattering by pixel selection. Without pixel selection, scattering would dominate at low acetylene concentrations [10].

In Fig. 3, a range of acetylene spectra obtained at low concentrations around the R9e line of the $\left(010(11)^{\wedge} 0\right)$ $\left(0000^{\wedge} 00^{\wedge} 0\right)$ band line is displayed. To reduce the effect of power fluctuations from the mid-IR source, each trace is an average of 10 identical sweeps that each includes all the wavenumbers, as indicated by the first two raw image blocks in Fig. 2. Furthermore, the background and part of the scattering are removed by subtraction of a reference spectrum acquired with a pure $\mathrm{N}_{2}$ gas flow. During the scan, dust particles occasionally passed through the beam, causing major single frame spikes in the signal due to scattering. These spikes have been removed by postprocessing before the spectra were generated, and finally to smooth out the graphs, a 10-point running average has been applied on the data. The peaks for the different concentrations do not overlap completely due to wavelength fluctuations of the mid-IR light source that origin from mode hops and instabilities. As this relates directly to the light source, it is relevant for both types of detectors. Overall, the measurements correspond well with the gas line simulation from the Hitran 2012 database [12], as seen from Fig. 3 . This simulation is generated with a Voigt line-shape function [7] with a half-width at half-maximum linewidth of $0.06 \mathrm{~cm}^{-1}$. This empirical approach includes contributions from Doppler, power, laser linewidth, and collisional broadening [7].

Across short scans, the phase-matching condition for the frequency upconversion can be regarded as constant, but for longer scans it is necessary to tune the

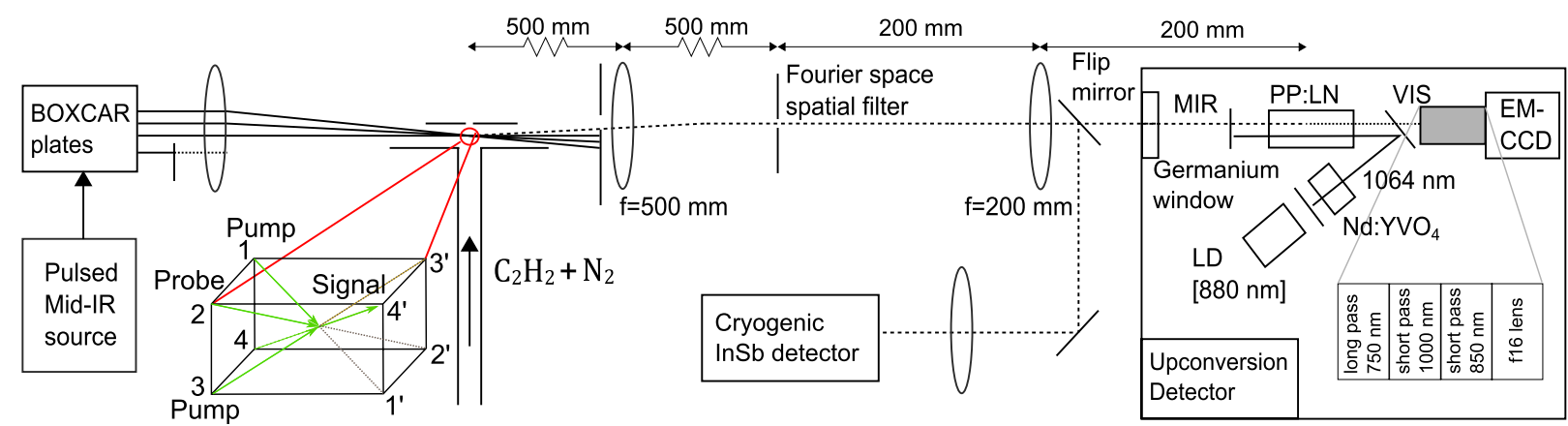

Fig. 1. Sketch of the full DWFM experimental setup consisting of a pulsed mid-IR light source, a set of BOXCAR plates that splits the beam into four, a gas tube with a diluted acetylene flow, and the choice of two detectors for the generated signal. 


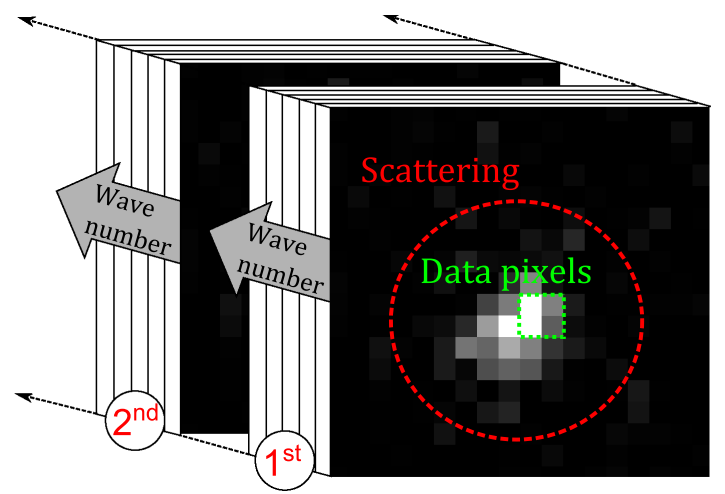

Fig. 2. Illustration of the raw data image stack that forms the basis for the measured spectra. Each point in a spectrum is the sum of the pixels in a single frame. The displayed frame is a section of the full image and recorded at an acetylene concentration of $6 \mathrm{ppm}$.

temperature continuously during the wavelength sweep. This has been demonstrated in [10].

The detected signal is given as photons per pixel per pulse and, in the following, the conversion from camera counts to detected photons is described. Specifically, the absolute calibration is computed from the camera counts by comparing the difference in camera counts with and without electron multiplication and the specified well depth of the CCD without electron multiplication. The dark current is assumed to be 0 , which is a good assumption given the dark current of the camera of $0.05 \mathrm{e} / \mathrm{pixel} / \mathrm{s}$ with an exposure time of $1 \mathrm{~ms}$. The photon number estimation is verified by computing the standard deviation of the camera counts from an unilluminated area of the CCD. This gives a reasonable estimate of the read noise measured in camera counts. From the conversion factor from well-depth estimation, this corresponds to a read noise of 0.5 e. The specified read noise from the CCD in electron multiplication mode is $<1 \mathrm{e}$, thus verifying the power calibration of the camera.

The total amount of detected photons is defined by the selected number of data pixels, in this case four, as shown in Fig. 2. Without spatial filtering and in the case

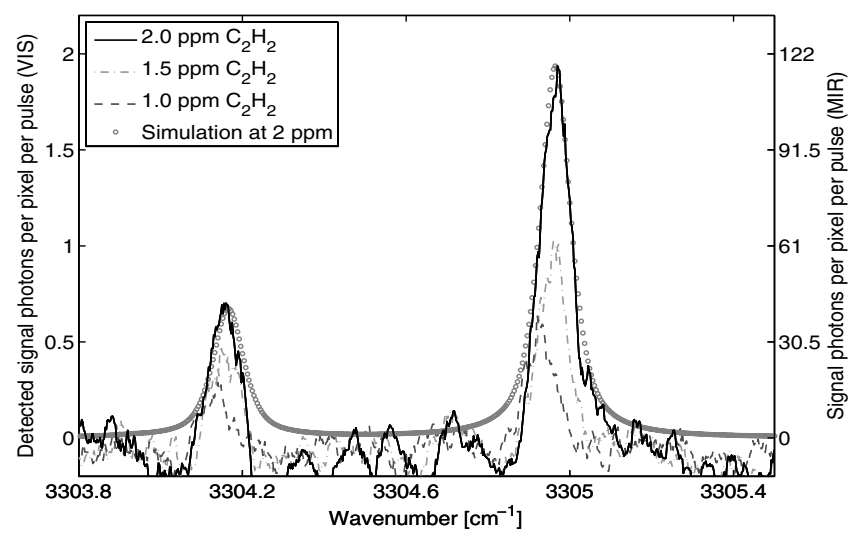

Fig. 3. Examples of spectra obtained at low acetylene concentrations. Each trace is the average of 10 scans and a running average of 10 points has been applied after the background obtained at $0 \mathrm{ppm}$ has been subtracted. The intensity values are computed from the camera specifications and the right axis is calculated from the inherent loss in the detector system. of no scattering, the data pixel area could be extended to include the whole signal area, thus increasing the signal intensity by a factor of approximately 4 . To give an understanding of the sensitivity of the upconversion detector in the mid-IR regime, the right $y$ axis in Fig. 3 describes the amount of incoming mid-IR signal photons, calculated from the estimated total power transmission efficiency:

$$
\eta=\eta_{\mathrm{CCD}} \cdot \eta_{\text {conv }} \cdot \eta_{\text {filters }}=0.22 \cdot 0.15 \cdot 0.5=0.017,
$$

where $\eta_{\mathrm{CCD}}$ and $\eta_{\text {conv }}$ are the quantum efficiencies of the camera and the nonlinear process, respectively, and $\eta_{\text {filters }}$ is the power fraction through the filter set described in Fig. 1.

The line integration of a single gas line, i.e., the area under the peak, obtained from a DWFM measurement is expected to have a quadratic dependence with the gas concentration under optically thin conditions [7]. This is supported by the plot in Fig. 4 , where the measured line integrated intensity of eight different acetylene concentrations from the upconversion detector and three from the InSb detector are plotted. The data acquired with the upconversion detector are fitted to the equation $y=a x^{k}$. A best fit procedure results in $k=1.94$, showing a good fit to the expected value of $k=2$. For the $12 \mathrm{ppm}$ concentration, there is a discrepancy for the InSb measurement as this point is close to the noise floor of the detector. The specific acetylene concentrations were calculated from the mass flowmeter readings and the gas concentration used. The error bars in Fig. 4 represent the standard deviation on the line integrated intensities obtained from 10 identical measurements.

The underlying spectra of the three lowest concentrations are shown in Fig. 3 . The horizontal lines in Fig. 4 show the standard deviation on the line integration of 10 identical measurements with pure nitrogen flow for the InSb and the upconversion detector. This we define as the detector noise level for each detector, and the possible detection limit is the intersection between this line and the quadratic dependency line. From this we can quantify that the upconversion detector has approximately 500 times better SNR than the cryogenic InSb detector. Due to the quadratic dependency, this

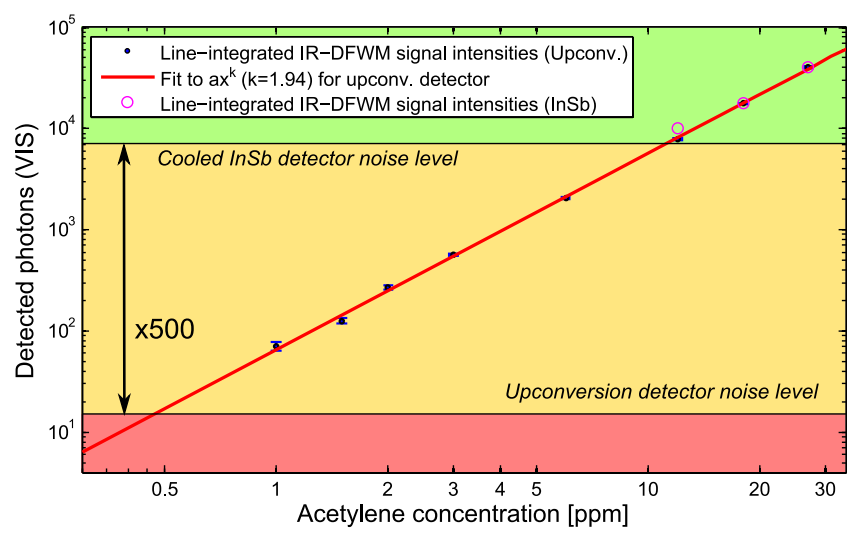

Fig. 4. Plot of the line integrated intensity of the R9e line of the $\left(010(11)^{\wedge} 0\right)-\left(0000^{\wedge} 00^{\wedge} 0\right)$ band line as a function of acetylene concentration. The noise levels are computed from the standard deviation on the noise in the two detectors. The error bars indicate the standard deviation on the signal intensities. 
Table 1. Standard Deviations of the Spectral Measurements Translated to Absolute Deviations of the Gas Concentrations

\begin{tabular}{lcc}
\hline $\begin{array}{l}\text { Concentration } \\
\mathrm{C}_{2} \mathrm{H}_{2}[\mathrm{ppm}]\end{array}$ & $\begin{array}{c}\text { Deviation [ppm] } \\
\text { (Upconv. detector) }\end{array}$ & $\begin{array}{c}\text { Deviation [ppm] } \\
\text { (InSb detector) }\end{array}$ \\
\hline 1.0 & $\pm 0.051(5.1 \%)$ & - \\
1.5 & $\pm 0.048(3.2 \%)$ & - \\
2.0 & $\pm 0.055(2.8 \%)$ & - \\
3.0 & $\pm 0.047(1.6 \%)$ & - \\
6.0 & $\pm 0.059(0.99 \%)$ & $\pm 0.43(3.6 \%)$ \\
12 & $\pm 0.12(1.0 \%)$ & $\pm 0.51(2.8 \%)$ \\
18 & $\pm 0.14(0.77 \%)$ & $\pm 0.33(1.2 \%)$ \\
27 & $\pm 0.24(0.91 \%)$ &
\end{tabular}

translates to a factor of more than 20 in improved detection limit compared to the InSb measurements at hand. Unfortunately, it was not possible to prepare acetylene concentrations below $1 \mathrm{ppm}$ in the current setup due to limitations in the mass flow controllers.

Table 1 shows how the standard deviation on the integrated peak intensities translates into deviations on the acetylene concentrations. From this we conclude that the upconversion technique is stable and generally performs well in DFWM gas detection. It seems that the precision is limited to $1 \%$, possibly limited by the laser power fluctuations described earlier.

It is possible to use an InSb detector with a smaller detector area and thus a smaller noise equivalent power (NEP), but this is not recommended due to beam wandering and fundamental alignment difficulties at these wavelengths.

To get a detailed understanding of the detector performance, a simple comparison of SNRs is not sufficient. From the experiments it was clear that the InSb detector is limited by the inherent NEP $\left(\mathrm{NEP}=3.0 \mathrm{pW} /(\mathrm{Hz})^{1 / 2}\right)$, where the upconversion detector is limited by the scattering. This indicates great potential for pushing the detection limit further down by further suppressing the residual scattering. This could be done by improving the anti-reflection coatings on the optical surfaces, by using higher grade optics in the system, or by more efficient filtering and screening. As the upconversion detector is ultimately limited by the signal shot noise, we expect the system to achieve detection limits that are 1 order of magnitude better in a scattering free setup.

A special feature of the upconversion detector is the inherent limited spectral and angular acceptance bandwidth. For the system at hand, the spectral acceptance bandwidth is calculated, from the Sellmeier equations, to be approximately $6 \mathrm{~nm}$ and the monochromatic field of view to be $16 \mathrm{mrad}$. In high-temperature measurements, e.g., under flame conditions, this implies that only a narrow part of the thermal noise is collected, providing a significant advantage compared to conventional cryogenic detectors that both have a large field of view and an acceptance bandwidth that is several orders of magnitude broader. We have demonstrated that the upconversion detector can be applied as an efficient upgrade to a state-of-the-art low-signal mid-IR measurement system. The implementation is a simple detector replacement and further allows for imaging and correspondingly improved spatial filtering. From the quantitative comparison, we can conclude that the upconversion system shows near-shot-noise-limited performance, while the $\mathrm{InSb}$ detector is limited to 500 times lower SNR. This presents possibilities for many potential applications in which the detection of low-level mid-IR signals is critical.

Part of this work was financed by the Swedish Research Council (VR), the Swedish Energy Agency through the Centre for Combustion Science and Technology (CECOST), and European Research Council Advanced Grant DALDECS.

\section{References}

1. A. Rogalski, Infrared Detectors (CRC Press, 2010).

2. J. S. Pelc, L. Ma, C. R. Phillips, Q. Zhang, C. Langrock, O. Slattery, X. Tang, and M. M. Fejer, Opt. Express 19, 21445 (2011).

3. J. S. Pelc, Q. Zhang, C. R. Phillips, L. Yu, Y. Yamamoto, and M. M. Fejer, Opt. Lett. 37, 476 (2012).

4. G.-L. Shentu, X.-X. Xia, Q.-C. Sun, J. S. Pelc, M. M. Fejer, Q. Zhang, and J.-W. Pan, Opt. Lett. 38, 4985 (2013).

5. J. S. Dam, P. Tidemand-Lichtenberg, and C. Pedersen, Nat. Photonics 6, 788 (2012).

6. Z. S. Li, M. Linvin, J. Zetterberg, J. Kiefer, and M. Aldén, Proc. Combust. Inst. 31, 817 (2007).

7. Z. W. Sun, Z. S. Li, B. Li, M. Aldén, and P. Ewart, Appl. Phys. B 98, 593 (2010).

8. M. R. McCurdy, Y. Bakhirkin, G. Wysocki, R. Lewicki, and F. K. Tittel, J. Breath Res. 1, 014001 (2007).

9. W. Rock, Y.-L. Li, P. Pagano, and C. M. Cheatum, Phys. Chem. A 117, 6073 (2013).

10. L. Høgstedt, J. S. Dam, A. Sahlberg, Z. S. Li, M. Aldén, C. Pedersen, and P. Tidemand-Lichtenberg, Proc. SPIE 8964, 89641H (2014).

11. Z. S. Li, M. Rupinski, J. Zetterberg, Z. T. Alwahabi, and M. Aldén, Chem. Phys. Lett. 407, 243 (2005).

12. L. S. Rothman, I. E. Gordon, Y. Babikov, A. Barbe, D. C. Benner, P. F. Bernath, M. Birk, L. Bizzocchi, V. Boudon, L. R. Brown, A. Campargue, K. Chance, E. A. Cohen, L. H. Coudert, V. M. Devi, B. J. Drouin, A. Fayt, J.-M. Flaud, R. R. Gamache, J. J. Harrison, J.-M. Hartmann, C. Hill, J. T. Hodges, D. Jacquemart, A. Jolly, J. Lamouroux, R. J. Le Roy, G. Li, D. A. Long, O. M. Lyulin, C. J. Mackie, S. T. Massie, S. Mikhailenko, H. S. P. Müller, O. V. Naumenko, A. V. Nikitin, J. Orphal, V. Perevalov, A. Perrin, E. R. Polovtseva, C. Richard, M. A. H. Smith, E. Starikova, K. Sung, S. Tashkun, J. Tennyson, G. C. Toon, Vl. G. Tyuterev, and G. Wagner, J. Quant. Spectrosc. Radiat. Transfer 130, 4 (2013). 